\title{
Agenesia renal unilateral e criptorquidismo ipsilateral em um felino: relato de caso
}

\author{
[Unilateral renal agenesis and ipsilateral cryptorchidism \\ in a cat: case report] \\ E.R. Santos ${ }^{1}$, N.S. Rosa $^{1}$, B.S. Barni ${ }^{1}$, M.P. Oliveira ${ }^{1}$, V.M.F. Camargo ${ }^{1}$, E.A. Contesini ${ }^{2}$ \\ ${ }^{1}$ Hospital de Clínicas Veterinárias - Universidade Federal do Rio Grande do Sul - Porto Alegre, RS \\ ${ }^{2}$ Faculdade de Veterinária - Universidade Federal do Rio Grande do Sul - Porto Alegre, RS
}

\begin{abstract}
RESUMO
A agenesia renal é uma afecção congênita rara na espécie felina, frequentemente associada a uma malformação reprodutiva. $\mathrm{O}$ presente trabalho relata o caso de um felino com agenesia renal unilateral associada a criptorquidismo ipsilateral, com ênfase no diagnóstico, tratamento e acompanhamento. $\mathrm{O}$ paciente foi conduzido ao Hospital de Clínicas Veterinárias da Universidade Federal do Rio Grande do Sul para avaliação de criptorquidismo. A agenesia renal foi um achado durante a ecografia abdominal do felino. Durante a laparotomia, foi confirmada a ausência do rim e ureter direito, hipertrofia do rim esquerdo e presença de um testículo ectópico. O paciente teve alta após a recuperação anestésica e se mantém clinicamente estável, transcorridos seis meses da cirurgia. A agenesia renal unilateral é uma condição compatível com a vida, contanto que o rim existente apresente funcionamento aceitável. Assim, sugere-se que a possibilidade de rim único em felinos criptorquidas deve ser investigada sempre que possível, tendo em vista a alta correlação entre essas malformações, e objetivando um acompanhamento da função renal do paciente ao longo da vida.
\end{abstract}

Palavras-chave: malformação renal, rim único, testículo, doenças congênitas

\begin{abstract}
Renal agenesis is a rare disorder in feline species, commonly associated with reproductive malformation. This study aims to report the case of a cat with unilateral renal agenesis combined with ipsilateral cryptorchidism, emphasizing the diagnosis, treatment and patient follow up. The patient was taken to the Veterinary Hospital of the Federal University of Rio Grande do Sul to evaluate the cryptorchidism. The renal agenesis was an incidental finding during the abdominal ultrasound. At laparotomy, the absence of the right kidney and ureter was confirmed, hypertrophy of the left kidney and the presence of an ectopic testicle were found. The patient was discharged after recovering from anesthesia and remains clinically stable six months after surgery. The unilateral renal agenesis is a life compatible condition as long as the existing kidney has an acceptable performance. Therefore, it is suggested that the chance of a single kidney in cats whit cryptorchidism should be investigated, given the correlation between these malformations, and aiming to monitor renal function throughout the life of the patient.
\end{abstract}

Keywords: renal malformation, single kidney, testicle, congenital diseases

\section{INTRODUÇÃO}

A agenesia renal unilateral é um defeito congênito bastante raro em pequenos animais. Configura-se na ausência completa de um dos rins, situação com a qual o animal pode viver de

Recebido em 19 de junho de 2014

Aceito em 3 de março de 2015

E-mail: eduardo.rosasantos@gmail.com forma satisfatória se existir um rim normal para assumir as funções. Em associação, há possibilidade de ocorrer agenesia ureteral ipsilateral e hipertrofia compensatória do rim remanescente (Fedalto, 2012). Devido à hipertrofia compensatória, a agenesia renal unilateral frequentemente não é notada, embora 
já tenha sido reportada em cães, gatos e humanos. Já a agenesia bilateral é sempre fatal. Nos casos em que o rim único apresenta-se hipoplásico ou displásico, o paciente pode desenvolver insuficiência renal e assim o diagnóstico é mais precoce (Narasimhan e Absar, 2010).

Normalmente a enfermidade está associada à malformação do trato reprodutivo, já que, durante o desenvolvimento embrionário, os rins, trato urinário e a maior parte dos órgãos reprodutivos surgem do mesoderma intermediário. A coexistência entre malformações urinárias e genitais tem sido identificada mais frequentemente em fêmeas do que em machos da espécie felina. (Chang et al., 2008; Narasimhan e Absar, 2010; Carvallo et al., 2012).

O criptorquidismo é a falha de um ou de ambos os testículos em descer e permanecer no saco escrotal por volta dos sete a oito meses de idade em felinos. Essa é a afecção congênita mais comum do sistema urogenital dessa espécie, ainda que a sua prevalência seja relativamente baixa, em torno de 1,3 a 3,8\% (Little, 2011).

Objetivou-se neste trabalho descrever um caso incomum, atendido no Hospital de Clínicas Veterinárias da Universidade Federal do Rio Grande do Sul (HCV-UFRGS), de um felino macho com agenesia renal unilateral $\mathrm{e}$ criptorquidismo ipsilateral, com ênfase no diagnóstico, tratamento e acompanhamento em curto prazo.

\section{CASUÍSTICA}

Um felino Himalaia de um ano de idade foi atendido no HCV-UFRGS com suspeita de testículo intra-abdominal. $\mathrm{Na}$ anamnese, a tutora não referia nenhuma alteração no desenvolvimento e comportamento do paciente. No exame físico geral, todos os parâmetros estavam dentro do esperado para a espécie e na palpação testicular apenas um testículo foi localizado na bolsa escrotal.

O felino foi encaminhado para ecografia, a fim de se avaliar a localização do testículo criptorquida. Durante o exame, foi visibilizado apenas um rim (topografia renal esquerda), com dimensões aumentadas $(5,1 \mathrm{~cm}$ em eixo longitudinal), contornos regulares, arquitetura, ecogenicidade e relação corticomedular preservados (Fig. 1).

Em topografia renal direita, foi localizada uma estrutura com contornos definidos e pouco regulares, heterogênea, delimitada, medindo cerca de $1,6 \mathrm{~cm}$, compatível com testículo incluso ou rim degenerado (Fig. 2). O testículo presente na bolsa escrotal apresentava rafe e ecotextura homogênea.

Além da ultrassonografia abdominal, foram solicitados hemograma, perfil bioquímico (alanina aminotransferase - ALT, creatinina, albumina) e teste para Vírus da Imunodeficiência Felina (FIV) e da Leucemia Felina (FeLV). No hemograma não houve alterações, enquanto no perfil bioquímico a ALT estava levemente elevada $(93,75 \mathrm{U} / \mathrm{L}$ - referência: $<83 \mathrm{U} / \mathrm{L})$ e a creatinina e albumina estavam dentro dos padrões normais para a espécie. Os snap testes para FIV e FeLV resultaram negativos. Com os resultados dos exames, o paciente foi encaminhado para orquiectomia e laparotomia para criptorquidectomia e avaliação do sistema urinário.

O paciente foi preparado de forma rotineira para a cirurgia. Para acessar a cavidade abdominal, foi realizada uma incisão pré-retro umbilical na linha alba. $O$ rim esquerdo $( \pm 5 \mathrm{~cm} \times 3 \mathrm{~cm}$, coloração normal), ureter esquerdo e bexiga urinária foram localizados. O testículo direito (hipotrofiado) foi encontrado em topografia mesogástrica direita (Fig. 3), pinçado, seccionado e ligado duplamente com náilon 3-0. Em região de rim direito não foi localizado o órgão nem o respectivo ureter, a adrenal ipsilateral estava presente e os vasos renais terminavam em fundo de saco cego. Os demais órgãos abdominais foram inspecionados e não apresentavam alteração. A parede muscular da cavidade abdominal foi suturada com náilon 3-0 (padrão simples contínuo), o subcutâneo reduzido com náilon 4-0 (padrão simples contínuo) e a dermorrafia realizada com náilon 4-0 (padrão intradérmico). 


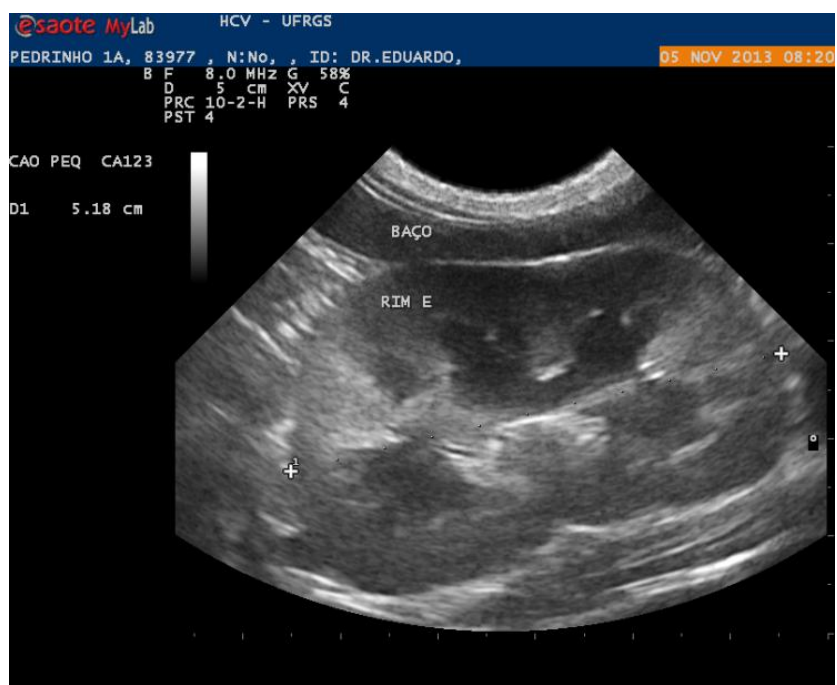

Figura 1. Imagem ecográfica do rim esquerdo (RIM E) com dimensões aumentadas $(5,1 \mathrm{~cm}$ no eixo longitudinal).

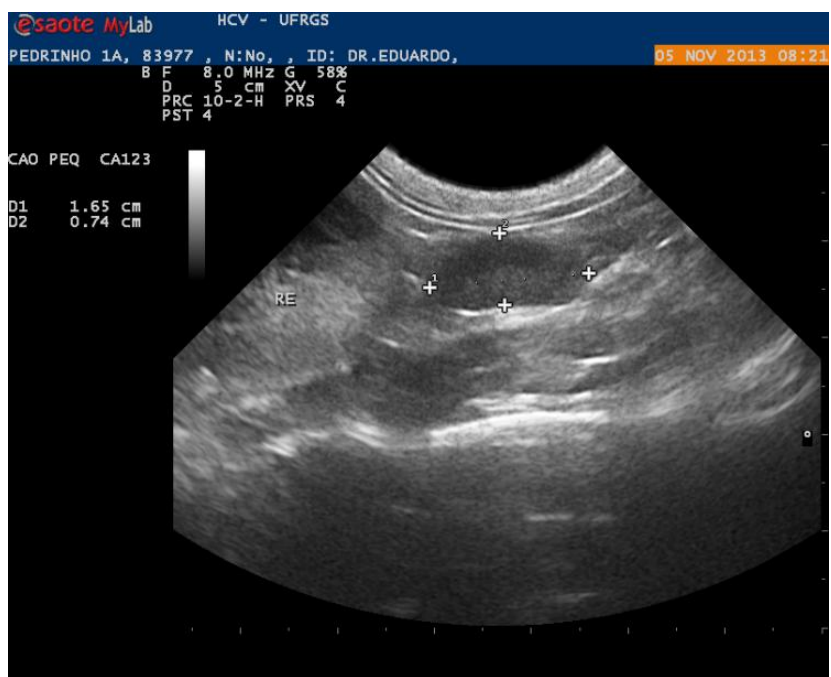

Figura 2. Imagem ecográfica da estrutura de 1,6cm compatível com testículo incluso ou rim degenerado.

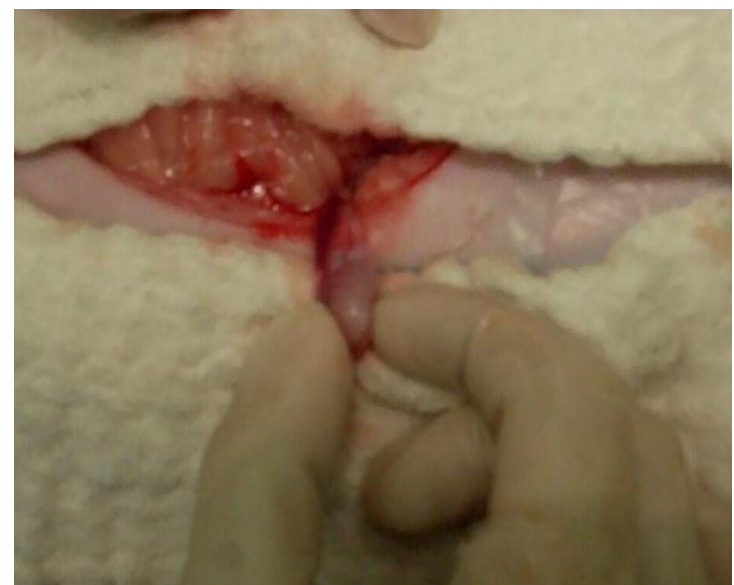

Figura 3. Testículo direito hipotrofiado em topografia mesogástrica direita (região do rim direito). 
A orquiectomia foi realizada em seguida empregando ligadura com náilon 3-0. A pele foi suturada com mesmo fio em pontos isolados simples. O paciente teve alta no mesmo dia após a recuperação anestésica. Os testículos foram encaminhados para análise histopatológica. O criptorquida apresentava degeneração testicular, media $1,4 \mathrm{~cm} \times 0,7 \mathrm{~cm}$, e os túbulos seminíferos estavam atrofiados com acentuada proliferação de células intersticiais (Leydig) e ausência de espermatozoides no epidídimo. Já o testículo presente na bolsa escrotal encontrava-se macroscopicamente normal $(2,3 \mathrm{~cm} \times 1,4 \mathrm{~cm})$, com espermatozoides no epidídimo e túbulos seminíferos íntegros.

Em dez dias, o paciente retornou ao hospital para a retirada de pontos. A incisão já estava cicatrizada, e o felino apresentava bom estado geral. Seis meses após o procedimento, foi realizado contato com a tutora. Esta informou que o paciente estava bem, urinando normalmente e com atividades normais.

\section{DISCUSSÃO}

O felino do presente caso possuía 12 meses de idade e, segundo Little (2011), aos oito meses os testículos já devem estar permanentemente no saco escrotal do gato. Diferentemente deste caso, ainda de acordo com a mesma autora, a localização inguinal é a mais comum no criptorquidismo, e tipicamente os testículos abdominais não apresentam espermatozoides quando avaliados histologicamente, devido às altas temperaturas a que estão expostos, tal qual o testículo abdominal aqui analisado.

O paciente não apresentava nenhum sinal clínico relacionado à agenesia renal, devido à compensação funcional pelo rim único. Segundo Bernstein et al. (2009), que diagnosticaram a mesma anomalia em um cão, os sinais clínicos quando presentes são relacionados à insuficiência renal. Os mesmos desenvolvem-se quando o rim remanescente não consegue absorver plenamente as funções do outro e falha em manter a homeostasia do organismo.

O diagnóstico de ausência renal pode ser estabelecido basicamente por duas formas. Em boa parte dos casos a malformação é um achado em um exame de imagem, como no felino aqui relatado, ou durante cirurgia da cavidade abdominal, como descrito por
Narasimhan e Abstar (2010), que diagnosticaram a agenesia renal em uma felina durante a ováriohisterectomia eletiva. Embora esses autores tenham realizado uma urografia excretora (póscirúrgica) para confirmação da malformação, esse exame não foi realizado no presente relato pelo fato de a ecografia deixar poucas dúvidas, pela necessidade de laparotomia para a criptorquidectomia e pelo risco de dano renal ocasionado pelo contraste intravenoso.

A ocorrência da malformação renal e uterina ou testicular, como neste caso, é explicada pelo desenvolvimento embrionário conjunto do sistema urinário e genital, o qual requer a diferenciação de dois tecidos derivados do mesoderma intermediário do embrião: o epitélio do ducto e o mesênquima nefrogênico. $\mathrm{O}$ primeiro dá origem ao trato genital, ureteres e rins, e o segundo sofre transformação para formar os néfrons. Desse modo, é importante suspeitar de agenesia renal na presença de malformações reprodutivas (Goo et al., 2008; Narasimhan e Abstar, 2010). Ainda, outras malformações urinárias podem estar presentes, como no felino descrito por Paradise e Clark (2013), em que havia criptorquidia associada a rim supranumerário ipsilateral malformado.

Em fêmeas felinas foram descritos dois casos de agenesia renal e aplasia de corno uterino ipsilateral por Chang et al. (2008) e Goo et al. (2008) que corroboram o caso aqui descrito. No trabalho de McIntyre et al. (2010), desenvolvido durante ovário-histerectomias eletivas, detectouse agenesia renal ipsilateral em quase $30 \%$ das felinas com anormalidades uterinas.

Já em humanos, vários casos de agenesia renal vêm sendo reportados (Argueso et al. 1992), mas, de acordo com os autores consultados neste relato, os casos em animais, especialmente em machos da espécie felina, são extremamente raros, principalmente quando associados a criptorquidismo ipsilateral. Há certa discordância entre médicos nefrologistas acerca do dano ao tecido renal ocasionado pelo aumento compensatório da filtração pelo rim único, o que poderia levar à destruição lenta e progressiva dos glomérulos remanescentes (Hedge e Coulthard, 2009). Extrapolando os dados para a veterinária, são recomendadas avaliações periódicas por toda a vida do animal com ausência de um rim, a fim de detectar precocemente qualquer alteração renal no rim existente. 


\section{CONCLUSÃO}

Sugere-se que todo felino criptorquida seja submetido à avaliação para detecção de agenesia renal, frente à possível associação dessas malformações. Essa condição, apesar de rara, tem um diagnóstico simples de se empregar. A ausência de um rim é compatível com a vida, desde que o rim presente exerça função satisfatória, e que seu desempenho seja acompanhado ao longo de toda a vida do animal.

\section{REFERÊNCIAS}

ARGUESO, L.R.; RITCHEY, M.L.; BOYLE, E.T. et al. Prognosis of patients with unilateral renal agenesis. Pediatr. Nephrol., v.6, p.412-416, 1992.

BERNSTEIN, M.; BERNSTEIN, K.K.; BRITTO, L.M.P.; LOLI, P.A. Agenesia renal unilateral em um cão: relato de caso. Medvep - Rev. Cient. Med. Vet. Peq. Anim. Anim. Est., v.7, p.140-142, 2009.

CARVALLO, F.R.; WARTLUFT, A.N.; MELIVILU, R.M. Unilateral uterine segmentary aplasia, papillary endometrial hyperplasia and ipsilateral renal agenesis in a cat. J. Feline Med. Surg., v.15, p.349-352, 2012.

CHANG, J.; JUNG, J-H.; YOON, J. et al. Segmental aplasia of the uterine horn with ipsilateral renal agenesis in a cat. J. Vet. Med. Sci., v.70, p.641-643, 2008.
FEDALTO, V. Agenesia renal em cão: relato de caso. 2012. Disponível em: <https://www.equalis.com.br/arquivos_fck_editor/tcc_ vanessafedalto.pdf>. Acessado em: 19 abr. 2014.

GOO, M-J.; WILlIAMS, B. H.; HONG, I-H. et al. Multiple urogenital abnormalities in a Persian cat. $J$. Feline Med. Surg., v.11, p.153-155, 2008.

HEDGE, S.; COULTHARD, M.G. Renal agenesis and unilateral nephrotomy: what are the risks of living with a single kidney? Pediatr. Nephrol., v.24, p.439446, 2009

LITTLE, S. Problems and clinical challenges. $J$. Feline Med. Surg., v.13, p.508-515, 2011.

MCINITYRE, R.L.; LEVY, J.K.; ROBERTS, J.F. et al. Developmental uterine anomalies in cats and dogs undergoing elective ovariohysterectomy. J. Am. Vet. Med. Assoc., v.237, p.542-546, 2010.

NARASIMHAN, T.; ABSAR, Y. Incidental finding of renal agenesis in a cat. 2010. Disponível em: $<\mathrm{http}: / /$ veterinarymedicine.dvm360.com/vetmed/Felin e+Center/ Incidenta-finding-of-renal-agenesis-in-acat/ArticleStandard/Article/detail/673419>. Acessado em: 19 abr. 2014.

PARADISE, D.; CLARK, D. Supernumerary kidney with ipsilateral cryptorchidism in a cat. J. Am. Anim. Hosp. Assoc., v.49, p.338-341, 2013. 